\title{
Paediatric severe asthma: a need for novel innate molecular phenotypes
}

\author{
Kristina Laubhahn $\mathbb{1}^{1,2}$ and Bianca Schaub ${ }^{1,2}$ \\ Affiliations: ${ }^{1}$ Pediatric Allergology, Dept of Pediatrics, Dr. von Hauner Children's Hospital, University Hospital, \\ LMU Munich, Munich, Germany. ${ }^{2}$ Member of German Center for Lung Research - DZL, LMU Munich, Munich, \\ Germany.
}

Correspondence: Bianca Schaub, University Children's Hospital Munich, Ludwig Maximilian University, LMU, Lindwurmstr. 4, 80377 Munich, Germany. E-mail: bianca.schaublamed.uni-muenchen.de

@ERSpublications

Molecular phenotyping and mainstay treatment of children with severe asthma is a great challenge and requires further in depth studies http://bit.ly/2ME9GQP

Cite this article as: Laubhahn K, Schaub B. Paediatric severe asthma: a need for novel innate molecular phenotypes. Eur Respir J 2019; 54: 1901459 [https://doi.org/10.1183/13993003.01459-2019].

Asthma is a heterogeneous chronic inflammatory disease of the airways with variety of different phenotypes and is most frequently classified according to clinical phenotypes into allergic and non-allergic asthma [1] or according to severity into intermittent, mild, moderate or severe asthma [2]. Asthma affects around $14 \%$ of children worldwide and different immune cells and inflammatory mediators play a role, leading to typical asthma pathophysiology [3]. Severe asthma is associated with high mortality, morbidity and healthcare expense [4]. It is generally characterised by poor asthma control with persistent symptoms and exacerbations despite high-doses of inhaled steroids (ICS), long-acting beta-agonists and/or biologicals [5]. Children with severe asthma are the most susceptible group of patients in urgent need for careful clinical phenotyping complemented by more defined molecular phenotypes, enabling more specific treatment regimens. However, molecular phenotyping of children with severe asthma is a great challenge based on low numbers of children, and the availability of sufficient and high-quality biomaterial taken in a standardised fashion.

In this issue of the European Respiratory Journal, the study by NAGAKUMAR et al. [6] addresses the important challenge of a refined definition of paediatric severe asthma in a small group of 30 children and their airway molecular phenotypes. Previous studies divided severe asthma into "difficult-to-treat asthma" (DA) and "severe therapy-resistant asthma" (STRA) [7]. DA is characterised by poor asthma control despite maximal prescribed therapy, including high doses of ICS and additional medications due to comorbidity. Poor control is most commonly due to poor or non-adherence to treatment, or adverse psychological and environmental factors. STRA is also characterised by persistent poor control despite high-dose therapy, yet optimal adherence, control of allergen and efficient inhaler techniques [8]. This study has now addressed several key issues for children with severe asthma, including clinical and molecular phenotyping. Of note, STRA is itself heterogeneous and different STRA phenotypes are being recognised [9]. This study focused on DA and STRA phenotypes, whereby STRA patients had no improved asthma control, characteristic persistent poor lung function, frequent attacks and multiple atopic sensitisations. Previous studies showed that early atopy is a major risk factor for severe childhood asthma, including skin prick test wheals to inhalant and food allergens as phenotypic characteristics of STRA [10]. While Nagakumar et al. [6] showed no significant differences in specific aeroallergen IgE, it is possible that information on early life sensitisation could help to define these groups at an early stage. Importantly, 
total serum IgE levels and sputum eosinophils were significantly higher in STRA compared to DA or control children. NAGAKUMAR et al. [6] emphasised that airway eosinophilia is very specific to STRA, but not to DA. While this clearly helps to distinguish these groups, it also tentatively indicates that an even more subtle classification of a "high and intermediate eosinophilic" group, as arising in sputum and/or blood, may be indeed possible. While currently hampered by a low number of children, further stratification in even more defined sub-phenotypes of STRA may be of immense pathophysiological interest, though clearly a challenge. Nevertheless, this effort, as reported in this study, is critical and requires broad support to identify the most specific therapy options for this vulnerable patient population.

Of importance, lung function tests did not divide the groups but were more comparable with normal lung function values, which Nagakumar et al. [6] asserted to the stable nature of the patients during the assessment. Teague et al. [11] supported this fact, showing that children with severe asthma do not always present with poor lung function, but rather with increased symptoms and exacerbations. Although challenging to design, an assessment of the group of STRA during the loss of symptom control at the time of exacerbation may reveal underlying molecular differences even more clearly.

Based on the key role of immune cells in asthma pathogenesis, NAGAKUMAR et al. [6] focused previously on the recently identified, rare lymphocyte population, namely innate lymphoid cells (ILCs) with a lack of antigen specificity (non-T, non-B lymphocytes). Similar to Th2 cells, type 2 ILCs (ILC2s) also produce interleukin (IL)4, IL5, and IL13, among others, and are involved in remodelling and inflammation [12]. Despite a relatively small sample size from an epidemiological point of view, this immune-based study generates fundamental results. NAGAKUMAR et al. [13] observed previously that ILC2s were present in the sputum of STRA children. Here, they focused on the differences between severe asthma phenotypes and showed for the first time that paediatric STRA had a distinct airway molecular phenotype with increased airway eosinophils and ILC2s, defined by IL13, compared to DA. Sputum CD4 ${ }^{+}$Th2 cells (CRTH2, IL4, IL13) were elevated in STRA versus control children, while IL17 ${ }^{+}$ cells were similar in all three groups.

The exact phenotype of sputum ILC2s from asthmatic children is unknown. In the literature, ILC2s are most prevalent in mucosal tissue such as the lung, and mainly defined as $\operatorname{Lin}^{\text {neg }}, \mathrm{CD} 127^{+}$cells. Nevertheless, there is so far no single marker that defines ILC2s at one site but is not present at any other site, making this population very heterogeneous [14]. The authors defined ILC2s by extracellular (CD127, CRTH2) and intracellular markers (IL13) of Lin $^{\text {neg }} \mathrm{CD} 45^{+}$cells. Most airway IL13 ${ }^{+} \mathrm{Lin}^{\text {neg }} \mathrm{CD} 45^{+}$cells were $\mathrm{CD}^{-} 7^{-}$(approximately 65\%), half of the cells were CRTH2 ${ }^{-}$and only $16 \%$ were double positive. These results may contribute to the understanding of ILC2 subtypes in severe asthma.

Besides lung mucosa, ILCs are present in the upper airway mucosa [12]. Due to technically demanding procedures in young children, the group was able to collect a low amount of sputum samples from a small number of children. Moreover, ILCs represent a rare population per se, allowing a limited number of functional experiments (limited markers, cultured ILC2 experiments in vitro from adults). Of note, developing techniques for analysis of low volume biosamples of nasal swabs or nasal brushings will help to disentangle the role of ILCs in the future and possibly provide information on additional critical cell subpopulations. This aspect and the fact that molecular phenotypes are most likely not characterised by imbalance of one but several cell subpopulations may be a relevant facet for further functional experiments, to improve our understanding of molecular phenotypes in severe asthma.

An open need is a better insight into the effect of the mainstay treatment of severe asthmatics, namely high dose steroids, on diverse cell populations. While steroids have been universally introduced [15], their specific impact on more recently defined cell populations, such as ILCs, is sparsely defined in both STRA and DA $[16,17]$.

In this context, NAGAKUMAR et al. [6] investigated the effect of systemic steroids on different ILC subtypes in induced sputum, and cultured PBMCs, showing reduced airway ILCs $\left(\mathrm{CRTH} 2^{+}\right.$and $\left.\mathrm{IL}_{1} 3^{+}\right)$and airway $\mathrm{CD}^{+}$T-cells $\left(\mathrm{CRTH}_{2}{ }^{+}\right.$and $\left.\mathrm{IL}_{1} 3^{+}\right)$after systemic steroid application (intramuscular triamcinolone) in vivo. Furthermore, an association with reduced exhaled nitric oxide, improved symptoms, and reduced exacerbations was observed in STRA patients. This was associated with reduced IL13 expression in sputum, possibly indicating sensitivity to systemic steroids.

However, these cells are higher in airways of children with STRA on maximal dose inhaled steroids. The results showed that ILCs are sensitive to systemic corticosteroids but not to inhaled steroids. Additionally, the frequency of $\mathrm{IL} 13^{+} \mathrm{CD} 4^{+}$T-cells and IL13 ${ }^{+} \mathrm{ILCs}\left(\mathrm{Lin}^{\text {neg }}\right.$ ) from STRA patients sensitised to house dust mite (HDM) were significantly reduced after HDM-stimulation and budesonide (steroid) versus HDM-extract stimulation in vitro. This indicates that ILCs themselves are steroid-sensitive. Other studies reported converse results, with steroid-resistance of ILC2s in patients with severe asthma [18], which may 
explain why not all asthma patients respond to corticosteroids. Exact dose, application route and different vulnerability for children and adults require further studies.

Although IL17 is linked to severe, steroid-resistant adult asthma, no association with clinical parameters was found in children with STRA in this study. Airway IL17 ${ }^{+}$ILCs were not increased in STRA versus DA or controls and remained elevated following steroid treatment in vivo and in vitro. Previously, ILC3s were mainly defined as IL17-producing cells. Nevertheless, ILCs can convert from one into another phenotype (plasticity), and recent data showed that ILC2s converted into IL17-producing ILC3 cells in patients with cystic fibrosis [19]. If the observation of NAGAKUMAR et al. [6] that sputum IL17 ${ }^{+}$ILCs have little functional impact for STRA and DA phenotypes is confirmed, their role for personalised therapy regimens needs to be scrutinised.

NAGAKUmar et al. [6] have set the scene, unravelling molecular mechanisms for these clinically well-defined though challenging children with STRA and DA. A few key issues require attention. The context of genetic or epigenetic risk factors and environment-driven modification of ILCs, e.g. by the microbiome, may confront researchers with issues of power and feasibility in this small group of children. Moreover, since the immune system in paediatric patients is constantly developing, initially well-defined and well-confined asthma phenotypes may not be stable and may change over time. Thus, plasticity not only on a cellular level, but most likely also "clinically", puts clinicians in the position of regular re-evaluation of not only clinical but also molecular modulation. Also, steady state and exacerbation times require close attention and re-adjustment of diagnostics and therapies, potentially distinct in nature. Putting this into practice will require regular and easily accessible biosamples of small paediatric patients, and the development of affordable technologies to analyse these, at least in specialised paediatric pulmonological centres. Nevertheless, close clinical follow-up, together with support for adherence measures and treating comorbidities will be indispensable.

Conflict of interest: K. Laubhahn has nothing to disclose. B. Schaub reports grants from DFG and EU, and personal fees for consultancy from GlaxoSmithKline, Sanofi and Novartis, outside the submitted work.

\section{References}

1 Raedler D, Ballenberger N, Klucker E, et al. Identification of novel immune phenotypes for allergic and nonallergic childhood asthma. J Allergy Clin Immunol 2015; 135: 81-91.

2 Baos S, Calzada D, Cremades L, et al. Biomarkers associated with disease severity in allergic and nonallergic asthma. Mol Immunol 2017; 82: 34-45.

3 Global Initiative for Asthma. Global Strategy for Asthma Management and Prevention. www.ginasthma.org Date last updated: November 19, 2018. Date last accessed: July 12, 2019.

4 Barry LE, Sweeney J, O'Neill C, et al. The cost of systemic corticosteroid-induced morbidity in severe asthma: a health economic analysis. Respir Res 2017; 18: 129.

5 Brown $\mathrm{T}$, Jones $\mathrm{T}$, Gove $\mathrm{K}$, et al. Randomised controlled trials in severe asthma: selection by phenotype or stereotype. Eur Respir J 2018; 52: 1801444.

6 Nagakumar P, Puttur F, Gregory LG, et al. Pulmonary type-2 innate lymphoid cells in paediatric severe asthma: phenotype and response to steroids. Eur Respir J 2019; 54: 1801809.

7 Lødrup Carlsen KC, Hedlin G, Bush A, et al. Assessment of problematic severe asthma in children. Eur Respir J 2011; 37: 432-440.

8 Hedlin G, Bush A, Lødrup Carlsen K, et al. Problematic severe asthma in children, not one problem but many: a GA2LEN initiative. Eur Respir J 2010; 36: 196-201.

9 Bossley CJ, Fleming L, Ullmann N, et al. Assessment of corticosteroid response in pediatric patients with severe asthma by using a multidomain approach. J Allergy Clin Immunol 2016; 138: 413-420.

10 Bonner K, Roberts G. Does allergy explain why some children have severe asthma? Clin Exp Allergy 2018; 48: 1594-1605.

11 Teague WG, Phillips BR, Fahy JV, et al. Baseline features of the Severe Asthma Research Program (SARP III) cohort: differences with age. J Allergy Clin Immunol Pract 2018; 6: 545-554.

12 van Crombruggen K, Taveirne S, Holtappels G, et al. Innate lymphoid cells in the upper airways: importance of CD117 and IL-1RI expression. Eur Respir J 2018; 52: 1800742.

13 Nagakumar P, Denney L, Fleming L, et al. Type 2 innate lymphoid cells in induced sputum from children with severe asthma. J Allergy Clin Immunol 2016; 137: 624-626.

14 Gury-BenAri M, Thaiss CA, Serafini N, et al. The spectrum and regulatory landscape of intestinal innate lymphoid cells are shaped by the microbiome. Cell 2016; 166: 1231-1246.

15 Chu EK, Drazen JM. Asthma: one hundred years of treatment and onward. Am J Respir Crit Care Med 2005; 171: 1202-1208.

16 Castanhinha S, Sherburn R, Walker S, et al. Pediatric severe asthma with fungal sensitization is mediated by steroid-resistant IL-33. J Allergy Clin Immunol 2015; 136: 312-322.

17 Petersen BC, Budelsky AL, Baptist AP, et al. Interleukin-25 induces type 2 cytokine production in a steroidresistant interleukin-17RB+ myeloid population that exacerbates asthmatic pathology. Nat Med 2012; 18: 751-758.

18 Liu S, Verma M, Michalec L, et al. Steroid resistance of airway type 2 innate lymphoid cells from patients with severe asthma: the role of thymic stromal lymphopoietin. J Allergy Clin Immunol 2018; 141: 257-268.

19 Golebski K, Ros XR, Nagasawa M, et al. IL-1 $\beta$, IL-23, and TGF- $\beta$ drive plasticity of human ILC2 $s$ towards IL-17-producing ILCs in nasal inflammation. Nat Commun 2019; 10: 2162. 